\title{
Correction to: miR-671-5p Attenuates Neuroinflammation via Suppressing NF-KB Expression in an Acute Ischemic Stroke Model
}

\author{
Ling Deng ${ }^{1,2} \cdot$ Yi Guo $^{3} \cdot$ Jingdong Liu ${ }^{1} \cdot$ Xuan Wang $^{1} \cdot$ Sha Chen $^{1} \cdot$ Qian Wang ${ }^{1} \cdot$ Jianyan Rao ${ }^{1} \cdot$ Yuchun Wang $^{1}$. \\ Tianrui Zuo ${ }^{1}$ - Qingwen Hu ${ }^{1} \cdot$ Xiahong Zhao $^{1} \cdot$ Zhi Dong $^{1}$ (I)
}

Published online: 12 August 2021

(c) Springer Science+Business Media, LLC, part of Springer Nature 2021

\section{Correction to: Neurochemical Research (2021) 46:1801-1813 https://doi.org/10.1007/s11064-021-03321-1}

The original version of the article, Fig. 4e hippocampus contains a copied-and-pasted error in the preparation for publication.

This has been corrected by publishing this corrigendum.
We have provided corrected version of Fig. 4.

The authors declare that this correction do not change the results or conclusions of this paper. We sincerely apologize for having this error in the article, and apologize for any inconvenience caused.

The original article can be found online at https://doi.org/10.1007/ s11064-021-03321-1.

Zhi Dong

100798@cqmu.edu.cn

1 College of Pharmacology, The Key Laboratory of Biochemistry and Molecular Pharmacology, Chongqing Medical University, Chongqing 400016, China

2 Library, Southwest Medical University, Luzhou 646000, Sichuan, China

3 Department of Radiology, Chongqing University Central Hospital, Chongqing 400014, China 


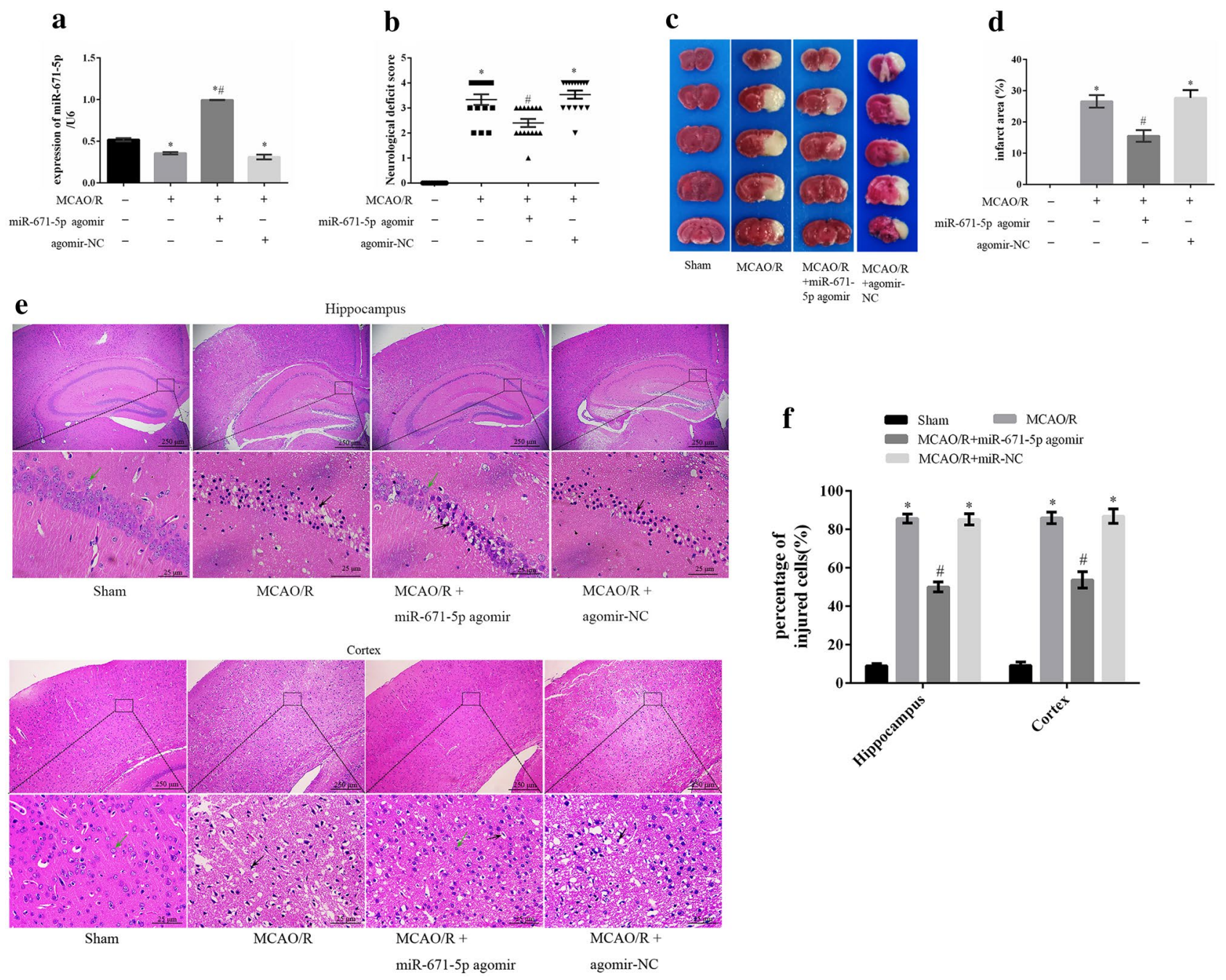

Fig. 4 Impact of miR-671-5P agomir in vivo. a Successful upregulation of miR-671-5P expression was achieved, and no significant effects imposed by the negative control were assessed via qRT-PCR $(n=6)$. b Neurological deficit scores $(n=6)$. c, d The area of infarc- tion were measured via TTC $(n=3)$. e, f H\&E staining was used to visualize the injured cells $(n=3)$; green arrow: normal cell, black arrow: injured cell with a reduced nucleus size. One-way ANOVA with Student's $t$ test, ${ }^{*} p<0.05$ vs sham, ${ }^{\#} p<0.05$ vs MCAO/R group

Publisher's Note Springer Nature remains neutral with regard to jurisdictional claims in published maps and institutional affiliations. 